\title{
Contents IMIA Yearbook of Medical Informatics 2019
}

\author{
President's Statement C. U. Lehmann \\ President's Statement \\ Editorial K. Fultz Hollis, L. F. Soualmia, B. Séroussi \\ Artificial Intelligence in Health Informatics: Hype or Reality? \\ 80th Birthday M. J. Ball, D. Bergemann, A. Hasman, R. Haux, A. T. McCray \\ On the 80th Birthday of Jan van Bemmel \\ IMIA Board Paper C. U. Lehmann, E. Borycki, K.-H. Cho, A.Espinosa, A. Geissbuhler, E. Huesing, P. Hurlen, \\ M. Kimura, G. Kovematchoua Tchuitcheu, A. Kushniruk, C. Lovis, D. Luna, A. Moen, \\ H.-A. Park, B. Séroussi, M. L. Silva, H. Takeda, P. Weber, J. Westbrook, S. Koch \\ On the Right to Science: Recommendations of Selection Criteria for IMIA Scientific Meetings \\ Keynote E. Coiera \\ The Price of Artificial Intelligence
}

Special Section:

Artificial Intelligence in Health:

New Opportunities, Challenges, and Practical Implications

Survey

F. Wang, A. Preininger

Al in Health: State of the Art, Challenges, and Future Directions

Working Group Contributions

L. Balkanyi, R. Cornet

The Interplay of Knowledge Representation with Various Fields of Arrificial Intelligence in Medicine

C. Kuziemsky, A. J. Maeder, O. John, S. B. Gogia, A. Basu, S. Meher, M. Ito

Role of Artificial Intelligence within the Telehealth Domain

H. Liyanage, S.-T. Liaw, J. Jonnagaddala, R. Schreiber, C. Kuziemsky, A. L. Terry, S. de Lusignan Artificial Intelligence in Primary Health Care: Perceptions, Issues, and Challenges

C. Paton, S. Kobayashi

An Open Science Approach to Artificial Intelligence in Healthcare

Synopsis G. Jackson, J. Hu

Artificial Intelligence in Health in 2018: New Opportunities, Challenges, and Practical Implications

Best Paper Selection Content summaries of:

Albers DJ, Levine ME, Stuart A, Mamykina L, Gluckman B, Hripcsak G. Mechanistic Machine Learning: How Data Assimilation Leverages Physiological Knowledge Using Bayesian Inference to Forecast the Future, Infer the Present, and Phenotype. J Am Med Inform Assoc 2018;25(10):1392-401

Lee J, Sun J, Wang F, Wang S, Jun CH, Jiang X. Privacy-Preserving Patient Similarity Learning in a Federated Environment: Development and Analysis. JMIR Med Inform 2018;6(2):e20

Oktay 0, Ferrante E, Kamnitsas K, Heinrich M, Bai W, Caballero J, Cook SA, de Marvao A, Dawes T, O'Regan DP, Kainz B, Glocker B, Rueckert D. Anatomically Constrained Neural Networks (ACNNs): Application to Cardiac Image Enhancement and Segmentation. IEEE Trans Med Imaging 2018;37(2):384-95 
Section 1: Health Information Management

Survey M. H. Stanfill, D. T. Marc

Health Information Management: Implications of Artificial Intelligence on Healthcare Data and Information Management

Synopsis M. Bloomrosen, E. S. Berner

Findings from the 2019 International Medical Informatics Association Yearbook Section on Health Information Management

Best Paper Selection Content summaries of:

Atutxa A, Pérez A, Casillas A. Machine learning approaches on diagnostic term encoding with the ICD for clinical documentation. IEEE J Biomed Health Inform 2018;22(4):1323-9

Cui L, Xie X, Shen Z. Prediction task guided representation learning of medical codes in EHR. J Biomed Inform 2018;84:1-10

Li F, Liu W, Yu H. Extraction of information related to adverse drug events from electronic health record notes: design of an end-to-end model based on deep learning. JMIR Med Inform 2018;6(4):e12159

Qiu JX, Yoon H-J, Fearn PA, Tourassi GD. Deep learning for automated extraction of primary sites from cancer pathology reports. IEEE J Biomed Health Inform 2018;22(1):244-51

Section 2: Human Factors and Organizational Issues

Survey P. Carayon, P. Hoonakkerk

Human Factors and Usability for Health Information Technology: Old and New Challenges

Synopsis S. Pelayo, Y. Senathirajah

Human Factors and Sociotechnical Issues

Best Paper Selection Content summaries of:

Couture B, Lilley E, Chang F, DeBord Smith A, Cleveland J, Ergai A, Katsulis Z, Benneyan J, Gershanik E, Bates DW, Collins SA. Applying user-centered design methods to the development of an mHealth application for use in the hospital setting by patients and care partners. Appl Clin Inform 2018 Apr;9(2):302-12

Miller A, Koola JD, Matheny ME, Ducom JH, Slagle JM, Groessl EJ, Minter FF, Garvin JH, Weinger MB, Ho SB. Application of contextual design methods to inform targeted clinical decision support interventions in sub-specialty care environments. Int J Med Inform 2018 Sep; 1 17:55-65

Tamblyn R, Winslade N, Lee TC, Motulsky A, Meguerditchian A, Bustillo M, Elsayed S, Buckeridge DL, Couture I, Qian CJ, Moraga T, Huang A. Improving patient safety and efficiency of medication reconciliation through the development and adoption of a computer-assisted tool with automated electronic integration of population-based community drug data: the RightRx project. J Am Med Inform Assoc 2018 May 1;25(5):482-95

Tscholl DW, Handschin L, Neubauer P, Weiss M, Seifert B, Spahn DR, Noethiger CB. Using an animated patient avatar to improve perception of vital sign information by anesthesia professionals. $\mathrm{Br} J$ Anaesth 2018 Sep;121(3):662-71 
Section 3: Clinical Information Systems

Survey C. Combi, G. Pozzi

Clinical Information Systems and Artificial Intelligence: Recent Research Trends

Synopsis W. O. Hackl, A. Hoerbst

Managing Complexity: From Documentation to Knowledge Integration and Informed Decision

Best Paper Selection Content summaries of:

Eichstaedt JC, Smith RJ, Merchant RM, Ungar LH, Crutchley P, Preoțiuc-Pietro D Asch DA,

Schwartz HA. Facebook language predicts depression in medical records. Proc Natl Acad Sci U S A 2018;115(44):11203-8

Parr SK, Shotwell MS, Jeffery AD, Lasko TA, Matheny ME. Automated mapping of laboratory tests to LOINC codes using noisy labels in a national electronic health record system database. J Am Med Inform Assoc 2018;25(10):1292-300

Xiao C, Ma T, Dieng AB, Blei DM, Wang F. Readmission prediction via deep contextual embedding of clinical concepts. PLoS One 2018;13(4):e0195024

Section 4: Sensor, Signal and Imaging

Informatics

Survey

C. Hoog Antink, S. Lyra, M. Paul, X. Yu, S. Leonhardt

A Broader Look: Camera-Based Vital Sign Estimation across the Spectrum

Synopsis $\quad$ W. Hsu, C. Baumgartner, T. Deserno

Advancing Artificial Intelligence in Sensors, Signals, and Imaging Informatics

Best Paper Selection Content summaries of:

Bandeira Diniz JO, Bandeira Diniz PH, Azevedo Valente TL, Corrêa Silva A, de Paiva AC,

Gattass $M$. Detection of mass regions in mammograms by bilateral analysis adapted to breast density using similarity indexes and convolutional neural networks. Comput Methods Programs Biomed 2018 Mar; 156:191-207

Lee H, Yune S, Mansouri M, Ki M, Tajmir SH, Guerrier CE, Ebert SA, Pomerantz SR, Romero JM, Kamalian S, Gonzalez RG, Lev MH, Do S. An explainable deep-learning algorithm for the detection of acute intracranial hemorrhage from small datasets. Nat Biomed Eng 2019 Mar;3(3):173-82

Samad MD, Ulloa A, Wehner GJ, Jing L, Hartzel D, Good CW, Williams BA, Haggerty CM, Fornwalt BK. Predicting survival from large echocardiography and electronic health record datasets: optimization with machine learning. JACC Cardiovasc Imaging 2019 Apr; 12(4):681-9

Vasilakakis MD, lakovidis DK, Spyrou E, Koulaouzidis A. DINOSARC: color features based on selective aggregation of chromatic image components for wireless capsule endoscopy. Comput Math Methods Med 2018 Sep 3;2018:2026962 
Section 5: Decision Support

Survey S. Montani, M. Striani

Artificial Intelligence in Clinical Decision Support: a Focused Literature Survey

Working Group Contribution

F. Magrabi, E. Ammenwerth, J. Brender McNair, N. F. De Keizer, H. Hyppönen, P. Nykänen, M. Rigby, P. J. Scott, T. Vehko, Z. S.-Y. Wong, A. Georgiou

Artificial Intelligence in Clinical Decision Support: Challenges for Evaluating Al and Practical Implications

Synopsis V. Koutkias, J. Bouaud

Contributions on Clinical Decision Support from the 2018 Literature

Best Paper Selection

\section{Content summaries of:}

Banerjee I, Gensheimer MF, Wood DJ, Henry S, Aggarwal S, Chang DT, Rubin DL. Probabilistic prognostic estimates of survival in metastatic cancer patients (PPES-Met) utilizing free-text clinical narratives. Sci Rep 2018 Jul 3;8(1):10037

Ray S, McEvoy DS, Aaron S, Hickman TT, Wright A. Using statistical anomaly detection models to find clinical decision support malfunctions. J Am Med Inform Assoc $2018 \mathrm{Jul}$ 1;25(7):862-71

Simon G, DiNardo CD, Takahashi K, Cascone T, Powers C, Stevens R, Allen J, Antonoff MB, Gomez D, Keane P, Suarez Saiz F, Nguyen Q, Roarty E, Pierce S, Zhang J, Hardeman Barnhill E, Lakhani K, Shaw K, Smith B, Swisher S, High R, Futreal PA, Heymach, Chin L. Applying Artificial Intelligence to address the knowledge gaps in cancer care. Oncologist 2018 Nov 16

Tsopra R, Lamy JB, Sedki K. Using preference learning for detecting inconsistencies in clinical practice guidelines: methods and application to antibiotherapy. Artif Intell Med 2018 Jul;89:24-33

Section 6: Knowledge Representation and Management

Survey

J. P. Bona, F. W. Prior, M. N. Zozus, M. Brochhausen Enhancing Clinical Data and Clinical Research Data with Biomedical Ontologies - Insights from the Knowledge Representation Perspective

Synopsis F. Dhombres, J. Charlet

Formal Medical Knowledge Representation Supports Deep Learning Algorithms, Bioinformatics

Pipelines, Genomics Data Analysis, and Big Data Processes

Best Paper Selection

Content summaries of:

Arguello Casteleiro M, Demetriou G, Read W, Fernandez Prieto MJ, Maroto N, Maseda

Fernandez D, Nenadic G, Klein J, Keane J, Stevens R. Deep learning meets ontologies: experiments to anchor the cardiovascular disease ontology in the biomedical literature. J Biomed Semantics 2018;9(1):13

Le KK, Whiteside MD, Hopkins JE, Gannon VPJ, Laing CR. Spfy: an integrated graph database for real-time prediction of bacterial phenotypes and downstream comparative analyses. Database (Oxford) 2018;2018:1-10

Osumi-Sutherland DJ, Ponta E, Courtot M, Parkinson H, Badi L. Using OWL reasoning to support the generation of novel gene sets for enrichment analysis. J Biomed Semantics 2018;9(1):10

Yu S, Ma Y, Gronsbell J, Cai T, Ananthakrishnan AN, Gainer VS, Churchill SE, Szolovits P, Murphy SN, Kohane IS, Liao KP, Cai T. Enabling phenotypic big data with PheNorm. J Am Med Inform Assoc 2018;25(1):54-60 
Section 7: Consumer Health Informatics

and Education

Survey N. Wickramasinghe

Essential Considerations for Successful Consumer Health Informatics Solutions

Working Group Contribution

K. Denecke, E. Gabarron, R. Grainger, S. T. Konstantinidis, A. Lau, 0. Rivera-Romero, T. Miron-Shatz, M. Merolli

Artificial Intelligence for Participatory Health: Applications, Impact, and Future Implications

Synopsis A. Y. S. Lau, P. Staccini

Arrificial Intelligence in Health: New Opportunities, Challenges, and Practical Implications

Content summaries of:

Abdellaoui R, Foulquié P, Texier N, Faviez C, Burgun A, Schück S. Detection of Cases of Noncompliance to Drug Treatment in Patient Forum Posts: Topic Model Approach. J Med Internet Res 2018;20(3):e85

Jones J, Pradhan M, Hosseini M, Kulanthaivel A, Hosseini M. Novel Approach to Cluster PatientGenerated Data Into Actionable Topics: Case Study of a Web-Based Breast Cancer Forum. JMIR Med Inform 2018;6(4):e45

Park A, Conway M, Chen AT. Examining Thematic Similarity, Difference, and Membership in Three Online Mental Health Communities from Reddit: A Text Mining and Visualization Approach. Comput Human Behav 2018 Jan; 78:98-112

Section 8: Bioinformatics and Translational Informatics

Survey

C. Overby Taylor, P. Tarczy-Hornoch

Personalized Medicine Implementation with Non-traditional Data Sources: A Conceptual

Framework and Survey of the Literature

Synopsis M. Smail-Tabbone, B. Rance

Contributions from the 2018 Literature on Bioinformatics and Translational Informatics

Lee SI, Celik S, Logsdon BA, Lundberg SM, Martins TJ, Oehler VG, Estey EH, Miller CP, Chien S, Dai J, Saxena A, Blau CA, Becker PS. A machine learning approach to integrate big data for precision medicine in acute myeloid leukemia. Nat Commun 2018 Jan;9(1):42

Mobadersany P, Yousefi S, Amgad M, Gutman DA, Barnholtz-Sloan JS, Velázquez Vega JE, Brat DJ, Cooper LAD. Predicting cancer outcomes from histology and genomics using convolutional networks. Proc Natl Acad Sci U S A 2018;115(13):E2970-E2979

Sengupta S, Sun SQ, Huang KL, Oh C, Bailey MH, Varghese R, Wyczalkowski MA, Ning J, Tripathi P, Mc Michael JF, Johnson KJ, Kandoth C, Welch J, Ma C, Wendl MC, Payne SH, Fenyö D, Townsend RR, Dipersio JF, Chen F, Ding L. Integrative omics analyses broaden treatment targets in human cancer. Genome Med 2018 Jul 27;10(1):60

Torshizi AD, Petzold LR. Graph-based semi-supervised learning with genomic data integration using condition-responsive genes applied to phenotype classification. J Am Med Inform Assoc 2018;25(1):99-108 
VI

(C) 2019

IMIA and Georg Thieme Verlag KG

Section 9: Clinical Research Informatics

Survey M.Cuggia, S. Combes

The French Health Data Hub and the German Medical Informatics Initiatives: Two National

Projects to Promote Data Sharing in Healthcare

Synopsis C. Daniel, D. Kalra

Clinical Research Informatics: Contributions from 2018

Best Paper Selection Content summaries of:

Brisimi TS, Chen R, Mela T, Olshevsky A, Paschalidis IC, Shi W. Federated learning of predictive models from federated Electronic Health Records. Int J Med Inform 2018 Apr; 1 12:59-67

Daniel C, Serre P, Orlova N, Bréant S, Paris N, Griffon N. Initializing a hospital-wide data quality program. The AP-HP experience. Comput Methods Programs Biomed 2018 Nov 9

Estiri H, Stephens KA, Klann JG, Murphy SN. Exploring completeness in clinical data research networks with DQe-c. J Am Med Inform Assoc 2018 Jan 1;25(1):17-24

Sylvestre E, Bouzillé G, Chazard E, His-Mahier C, Riou C, Cuggia M. Combining information from a clinical data warehouse and a pharmaceutical database to generate a framework to detect comorbidities in electronic health records. BMC Med Inform Decis Mak 2018 Jan 24;18(1):9

Section 10: Natural Language Processing

Survey M. Conway, M. Hu, W. W. Chapman

Recent Advances in Using Natural Language Processing to Address Public Health Research Questions Using Social Media and Consumer- Generated Data

Synopsis N. Grabar, C. Grouin, A Year of Papers Using Biomedical Texts: Findings from the Section on Natural Language Processing of the IMIA Yearbook

Best Paper Selection Content summaries of:

Jing B, Xie P, Xing E. On the automatic generation of medical imaging reports. Proc of ACL 2018. Melbourne, Australia; 2018. p. 2577-86

Moradi M. CIBS: A biomedical text summarizer using topic-based sentence clustering. J Biomed Inform 2018:88:53-61

Section 11: Public Health and Epidemiology Informatics

Survey

A. Rodríguez-González, M. Zanin, E. Menasalvas-Ruiz

Public Health and Epidemiology Informatics: Can Artificial Intelligence Help Future GlobalChallenges?

An Overview of Antimicrobial Resistance and Impact of Climate Change in Disease Epidemiology

Synopsis R. Thiébaut, S. Cossin

Artificial Intelligence for Surveillance in Public Health

Best Paper Selection Content summaries of:

Arsevska E, Valentin S, Rabatel J, de Goër de Hervé J, Falala S, Lancelot R, Roche M. Web monitoring of emerging animal infectious diseases integrated in the French Animal Health Epidemic Intelligence System. PLoS One 2018 Aug 3;13(8):e0199960

Effland T, Lawson A, Balter S, Devinney K, Reddy V, Waechter H, Gravano L, Hsu D. Discovering foodborne illness in online restaurant reviews. J Am Med Inform Assoc 2018 Dec 1;25(12):1586-92

Wakamiya S, Kawai Y, Aramaki E. Twitter-Based Influenza Detection After Flu Peak via Tweets With Indirect Information: Text Mining Study. JMIR Public Health Surveill 2018 Sep 25;4(3):e65 
Section 12: Cancer Informatics

\section{Synopsis J. L. Warner, D. A. Patt}

Cancer Informatics in 2018: The Mysteries of the Cancer Genome Continue to Unravel, Deep Learning Approaches the Clinic, and Passive Data Collection Demonstrates Utility

Content summaries of:

\section{Best Paper Selection}

Bailey MH, Tokheim C, Porta-Pardo E, Sengupta S, Bertrand D, Weerasinghe A, Colaprico A,

Wendl MC, Kim J, Reardon B, Ng PK, Jeong KJ, Cao S, Wang Z, Gao J, Gao Q, Wang F, Liu

EM, Mularoni L, Rubio-Perez C, Nagarajan N, Cortés-Ciriano I, Zhou DC, Liang WW, Hess

JM, Yellapantula VD, Tamborero D, Gonzalez-Perez A, Suphavilai C, Ko JY, Khurana E, Park PJ, Van Allen EM, Liang H; MC3 Working Group; Cancer Genome Atlas Research Network, Lawrence MS, Godzik A, Lopez-Bigas N, Stuart J, Wheeler D, Getz G, Chen K, Lazar AJ, Mills GB, Karchin R, Ding L. Comprehensive characterization of cancer driver genes and mutations. Cell 2018 Apr 5;173(2):371-385.e18

Hosny A, Parmar C, Coroller TP, Grossmann P, Zeleznik R, Kumar A, Bussink J, Gillies RJ, Mak $\mathrm{RH}$, Aerts HJWL. Deep learning for lung cancer prognostication: A retrospective multi-cohort radiomics study. PLoS Med 2018 Nov 30;15(11):e1002711

Low CA, Dey AK, Ferreira D, Kamarck T, Sun W, Bae S, Doryab A. Estimation of symptom severity during chemotherapy from passively sensed data: Exploratory study. J Med Internet Res 2017 Dec 19;19(12):e420

Tamborero D, Rubio-Perez C, Deu-Pons J, Schroeder MP, Vivancos A, Rovira A, Tusquets I, Albanell J, Rodon J, Tabernero J, de Torres C, Dienstmann R, Gonzalez-Perez A, Lopez-Bigas

$\mathrm{N}$. Cancer Genome Interpreter annotates the biological and clinical relevance of tumor alterations. Genome Med 2018 Mar 28;10(1):25

\section{A. Kulikowski}

Beginnings of Artificial Intelligence in Medicine (AIM): Computational Artifice Assisting

Scientific Inquiry and Clinical Art - with Reflections on Present AIM Challenges

E. H. Shortliffe

Artificial Intelligence in Medicine: Weighing the Accomplishments, Hype, and Promise 
Information on IMIA Welcome to IMIA 263

Honorary Fellows 266

$\begin{array}{ll}\text { IMIA Member Societies } & 267\end{array}$

Institutional Members 268

Addresses of IMIA Member Societies 270

Information on IMIA Regions Information on APAMI (Asia Pacific Association for Medical Informatics) 286

Information on Helina (African Region) 291

Information on MENAHIA (Middle East and North African Association for Health Informatics) 292

Information on NAMI (North-American Medical Informatics) 297

Information on EFMI (European Federation For Medical Informatics) 301

$\begin{array}{llr}\text { Miscellaneous } & \text { Contributors } & 307\end{array}$

Reviewers 308

$\begin{array}{ll}\text { IMIA Yearbook Special Topics } & 309\end{array}$ 